\title{
A Directional Approach for Testing Homogeneity of Inverse Gaussian Scale-Like Parameters
}

\author{
Wong $\mathrm{ACM}^{1 *}$ and Zhang $\mathrm{S}^{2}$ \\ ${ }^{1}$ Department of Mathematics and Statistics, York University, Canada \\ ${ }^{2}$ Department of Statistics and Actuarial Science, University of Waterloo, Canada
}

Submission: May 15, 2017; Published: October 04, 2017

*Corresponding author: Wong ACM, Department of Mathematics and Statistics, York University, Canada, Email: august@yorku.ca

\section{Abstract}

In recent years, likelihood-based higher order asymptotic methods have been developed to obtain inference for a scalar parameter of interest. In this paper, a directional test for a vector parameter of interest from a natural exponential family model is derived based on the likelihood-based higher order asymptotic methods. The proposed method is then applied to obtain p-value for testing homogeneity of inverse Gaussian scale-like parameters. Example and simulation results illustrate that the proposed method works almost perfectly.

Keywords: Canonical parameter; Log-likelihood ratio statistic; Saddle point method; Score variable; Sufficient statistic

\section{Introduction}

Let $y_{1}, \cdots y_{n}$ be $\mathrm{n}$ identical and independently distributed random variables from a natural exponential family model with a k-dimensional canonical parameter $\varphi$ and $\mathrm{k}<\mathrm{n}$. Then the joint density is

$$
f(y ; \varphi)=\exp \left\{\varphi^{T} u-K(\varphi)\right\} h(y)
$$

And the corresponding log-likelihood function is

$$
\ell(\varphi)=\ell(\varphi ; y)=\ell(\varphi ; u)=\varphi^{T} u-K(\varphi)
$$

Where $y=\left(y_{1}, \ldots \ldots \ldots, y_{n}\right)^{T} \quad$ and $\quad u=u(y)=\left(u_{1}(y), \ldots \ldots ., u_{k}(y)\right)^{T} \quad$ is a sufficient statistic. Denote $u^{0}=u\left(y^{0}\right)$ and $s=u-u^{0}$ where $y^{0}$ is an observed data point. Then (2) can be rewritten as

$$
\begin{aligned}
& \ell(\varphi)=\ell(\varphi ; s)=\varphi^{T}\left(u-u^{0}\right)+\ell\left(\varphi ; u^{0}\right)=\varphi^{T} s+\ell^{0}(\varphi) \\
& \text { Where } \ell^{0}(\varphi)=\ell(\varphi ; s=0)=\ell\left(\varphi ; u=u^{0}\right) .
\end{aligned}
$$

In many applications, the parameter of interest is only a subset of the original parameters. Therefore, let $\varphi=\left(\psi^{T}, \lambda^{T}\right)^{T}$ where is a d-dimensional parameter of interest and $\lambda$ is a $(k-d)$-dimensional nuisance parameter. Then the log-likelihood function in (3) can be rewritten as

$$
\ell(\varphi)=\ell(\varphi ; s)=\psi^{T} s_{1}+\lambda^{T} s_{2}+\ell^{0}(\psi, \lambda) .
$$

It is well-known that the conditional density of $S_{1}$ given $S_{1}=s_{2}$ is also a natural exponential family model and it has the form

$$
f\left(s_{1} \mid s_{2} ; \psi\right)=\exp \left\{\psi^{T} s_{1}-K_{1}(\psi)\right\} h_{1}\left(s_{1}, s_{2}\right)(5)
$$

Which depends only on the parameter of interest $\psi$. Hence, according to Lehmann \& Romano [1], inference concerning $\psi$ will be based on the exact expression of (5). However, in general, both $K_{1}(\psi)$ and $h_{1}\left(s_{1}, s_{2}\right)$ are rarely explicitly available. Thus asymptotic methods are employed to obtain inference concerning $\psi$.

Two commonly used asymptotic methods are based on the asymptotic distributions of the maximum likelihood estimator and the log-likelihood ratio statistic. Let $\hat{\varphi}=\left(\hat{\psi}^{T}, \hat{\lambda}^{T}\right)$ be the overall maximum likelihood estimate, which is obtained by solving

$$
\left.\frac{\partial \ell(\varphi ; s)}{\partial \varphi}\right|_{\hat{\varphi}}=0
$$

Or, equivalently, by solving the score equation from (3)

$$
s=-\ell_{\varphi}^{0}(\hat{\varphi} ; s)=-\left.\frac{\partial \ell^{0}(\varphi ; s)}{\partial \varphi}\right|_{\hat{\phi}}=0 .
$$

Then, with the regularity conditions stated in DiCiccio et al. [2], $\hat{\varphi}$ is asymptotically distributed as a k-dimensional normal distribution with mean and variance-covariance matrix $\operatorname{var}(\hat{\varphi})$ where $\operatorname{var}(\hat{\varphi})$ can be approximated by either the inverse of the Fisher's expected information matrix, $\left\{E\left[-\frac{\partial^{2} \ell(\varphi ; s)}{\partial \varphi \partial \varphi^{T}}\right]\right\}^{-1}$, or the inverse of the observed information matrix

$$
\left\{j_{\varphi \varphi}(\hat{\varphi} ; s)\right\}^{-1}=\left\{-\left.\frac{\partial^{2} \ell(\varphi ; s)}{\partial \varphi \partial \varphi^{T}}\right|_{\hat{\varphi}}\right\}^{-1} .
$$

Since $\psi=(\underline{1}, \underline{0}) \varphi$ where $\underline{1}$ is a $(1 X d)$ vector of 1 and $(1 \times(k-d))$ 
vector of 0 , we have $\hat{\psi}$ is asymptotically distributed as a d-dimensional normal distribution with mean $\psi$ and variance covariance matrix $\operatorname{var}(\hat{\psi})=(1, \underline{0}) \operatorname{var}(\hat{\varphi})(\underline{1}, \underline{0})^{T} ;$ or equivalently $q(\psi)=(\hat{\psi}-\psi)^{T} \operatorname{var}(\hat{\psi})(\hat{\psi}-\psi)$ is a asymptotically distributed as a chi-square distribution with d degrees of freedom, $\chi_{d}^{2}$. Hence, the for testing

$$
H_{0}: \psi=\psi_{0} \quad \text { vs } H_{a}: \psi \neq \psi_{0}
$$

Is approximately $P\left(\chi_{d}^{2}>q\left(\psi_{0}\right)\right)$. Alternatively, let $\tilde{\varphi}=\hat{\varphi}_{\varphi}=\left(\psi^{T}, \hat{\lambda}_{\psi}^{T}\right)^{T}$ be the constrained maximum likelihood estimate which can be obtained by solving

$$
\left.\frac{\partial \ell(\varphi ; s)}{\partial \lambda}\right|_{\hat{\psi}_{v}}=0
$$

For each $\psi$. Then, again with the regularity conditions stated in DiCiccio et al. [2], the log-likelihood ratio statistic

$$
w(\psi)=2\left[\ell(\hat{\varphi} ; s)-\ell\left(\hat{\varphi}_{\psi} ; s\right)\right]
$$

Is also asymptotically distributed as $x_{d}^{2}$. The corresponding p-value is approximately $P\left(\chi_{d}^{2}>w\left(\psi_{0}\right)\right)$.

The above two asymptotic methods are known to be not very accurate especially when the sample size is small [3]. In recent years, various higher-order asymptotic methods have been proposed to obtain more accurate results. In particular, the saddlepoint method discussed in Daniels [4] and some of the associated methods, have generated lots of discussions in statistics literature. Nevertheless, most of the researches in these higher-order asymptotic methods, such as the Lugannani and Rice formula, Lugannani \& Rice [5], and the $r^{*}$ formula, Barndor-Nielsen [6,7], can only be applied to obtain inference for a scalar parameter of interest [8]. For a vector parameter of interest, only limited literature exists. For example, Skovgaard [9] derived a test statistic that was similar to the $r^{*}$ formula for a vector parameter of interest, and based on the idea from Fraser and Massam [10], Davision et al. [11] derived a directional test when the model is a linear exponential family model.

Note that the method by Skovgaard [9] attempts to correct the likelihood ratio statistic everywhere in the parameter space and, in general, is a relatively complicated method except for some special cases as those discussed in Skovgaard [9]. The proposed directional method, on the other hand, is designed to capture the alternative hypothesis suggested by the observed data. In Section 2, the directional test by Davison et al. [11] is discussed. It is then applied to testing homogeneity of the scalelike parameters of the inverse Gaussian distribution in Section 3. Numerical results are reported in Section 4 to demonstrate the accuracy of the proposed method. Some concluding remarks are recorded in Section 5 [12].

\section{Main result}

Fraser \& Massam [13] derived a directional test as an alternate way for testing a vector parameter of interest. In the context of marginal inference for regression parameters in nonnormal linear models, this test avoids the computation for the high-dimensional marginal density by replacing them with a one-dimensional conditional density. In this section, we applied the methodology to the natural exponential family model with a subset of the canonical parameter being the parameter of interest.

For a natural exponential family model with canonical parameter $\varphi=\left(\psi^{T}, \lambda^{T}\right)^{T}$, as discussed in Section 1, inference concerning will be based on the conditional density given in (5), which, in general, will not be explicitly available. Skovgaard [14] proposed a double saddlepoint method to approximate (5). On the other hand, Fraser et al. [12] proposed a sequential saddlepoint method to approximate the conditional likelihood function for and, hence, by normalizing the conditional likelihood function, we have

$$
\hat{f}(s ; \psi)=c \exp \left\{\ell\left(\hat{\varphi}_{\psi} ; s\right)-\ell(\hat{\varphi} ; s)\right\}\left|j_{\varphi \varphi}(\hat{\varphi} ; s)\right|^{-1 / 2} s \in L^{0}
$$

where $\mathrm{c}$ is the normalizing constant, $L^{0}$ is a d-dimensional plane defined by $s_{2}=0, \quad \hat{\varphi}$ is the overall maximum likelihood estimate of $\hat{\varphi} \hat{\varphi}_{\psi}$ is the constrained maximum likelihood estimate of $\hat{\varphi}$ for a fixed, $\psi$ and $\left|j_{\varphi \varphi}(\hat{\varphi} ; s)\right|$ is the determinant of the observed information matrix evaluated at the overall maximum likelihood estimate. Note that (7) is an approximation of (5) with the conditioning being implicitly accomodated by taking a "slice" through the full density. In other words, we are taking the full density but constraining s to lie $S$ when $\varphi=\hat{\varphi}_{\psi}^{0}$, be . $L^{\dagger}$ Note that

$$
s_{\psi}=-\ell_{\varphi}^{0}\left(\hat{\varphi}_{\varphi}^{0}\right)
$$

It is clear that $L^{\dagger}$ is in $L^{0}$ and $s_{\psi}$ depends on the observed data $y^{0}$. This line, $L^{\dagger}$ be parameterized by

$$
s(t)=s_{\psi}+t\left(s^{0}-s_{\psi}\right)=(1-t) s_{\psi} t \in \mathfrak{R}
$$

And, thus, $\hat{\varphi}$ in (7) varies with $\mathrm{s}(\mathrm{t})$. As $t$ increases, it traces a curve in the parameter space that passes through $\hat{\varphi}_{\psi}^{0}$. In particular, when $t=0$, it passes through $\hat{\varphi}_{\psi}^{0}$, and when $t=1$, it passes through $\hat{\varphi}^{0}$. Hence, by explicitly indicating that $\hat{\varphi}$ varies with $s(t)$, the saddlepoint approximation of (7) constrained to $L^{\dagger}$ can be written as

$$
h(t ; \psi)=\hat{f}(s(t) ; \psi)=c \exp \left\{\ell\left[\hat{\varphi}_{\psi}^{0} ; s(t)\right]-\ell[\hat{\varphi}(s(t)) ; s(t)]\right\}\left|j_{\varphi \varphi}[\hat{\varphi}(s(t)) ; s(t)]\right|^{-1 / 2} .
$$

To measure departure from the null hypothesis given in (6) along $L^{\dagger}$, we can use the conditional distribution of $\|s\|$ given the unit vector $a=s /\|s\|$. this joint density of $(\|s\|, a)$ can be obtained by change of variable from s to $(\|s\|, a)$.Then the marginal density of a can be obtained from integration and hence the conditional density is available. The most difficult part of the calculation is to obtain the Jacobian of the transformation. However, in Fraser \& Massam [10], they have calculated the Jacobian to be proportional to $t^{d-1}$. Therefore,

$$
t^{d-1} h(t ; \psi) \alpha \mathrm{t}^{d-1} \exp \left\{\ell\left[\hat{\varphi}_{\psi}^{0} ; s(t)\right]-\ell[\hat{\varphi}(s(t)) ; s(t)]\right\}\left|j_{\varphi \varphi}[\hat{\varphi}(s(t)) ; s(t)]\right|^{-1 / 2} .
$$

As in Fraser \& Massam [10], the p-value for testing the hypothesis in (6) can be viewed as the probability that $s(t)$ is as far or farther from $S_{\psi}$ than the observed value 0 . This p-value 
is referred to as the directed p-value, and mathematically, it can be calculated by

$$
P\left(\psi_{0}\right)=\frac{\int_{1}^{t_{\max }} t^{d-1} h\left(t ; \psi_{0}\right) d t}{\int_{0}^{t_{\max }} t^{d-1} h\left(t ; \psi_{0}\right) d t}
$$

where $t_{\max }$ is the is the largest value of $t$ for which the maximum likelihood estimator corresponding to $s(t)$ exists.

\section{Application to testing homogeneity of inverse Gaussian scale-like parameters}

The two-parameter inverse Gaussian distribution, $I G(\mu, \lambda)$ has density

$$
f(y ; \mu, \lambda)=\left(\frac{\lambda}{2 \pi y^{3}}\right)^{1 / 2} \exp \left\{-\frac{\lambda}{2 \mu^{2} y}(y-\mu)^{2}\right\} y>0
$$

where $\mu>0$ is the mean parameter and $\lambda>0$ is a scale-like parameter. Note that the $I G(\mu, \lambda)$ distribution belongs to the exponential family model with canonical parameters $\left(\frac{\lambda}{2 \mu^{2}}, \frac{\lambda}{2}\right)$.

This distribution is widely used to model positive right-skewed data. Doksum \& Hoyland [15], Seshadri $[16,17]$ and Takagi et al. [18] discussed applications of this distribution in accelerated life testing model analysis, survival data analysis and in occupational hygiene data analysis, respectively. Chhikara \& Folks [19] examined the equality of means problem from $k$ samples of independent $I G\left(\mu_{i}, \lambda_{j}\right)$ distributions, where $i=1, \ldots . ., k$. With the assumption that the scale-like parameters are the same, they derived the ANOVA F-test. Hence, testing

$$
H_{0}: \lambda_{1}=\ldots . .=\lambda_{k} \text { vs } H_{a}: \lambda_{i} \neq \lambda_{j} \text { for some } i \neq j
$$

Is of special interest. For testing the above hypothesis, Chhikara \& Folks [19] and also Chang et al. [20] discussed the likelihood ratio test, and Wong [21] proposed a saddlepoint approach. For the rest of this section, the directional test approach discussed in Section 2 is applied to this problem. Let $Y_{i 1}, \ldots . . Y_{i n i}$ be $n i$ independent random variables from $I G\left(\mu_{i}, \lambda_{j}\right)$ distribution for $i=1, \ldots ., k$. Moreover, assume these $\mathrm{k}$ inverse Gaussian distributions are independently distributed. Hence, the log-likelihood function is

$$
l(\theta)=\frac{1}{2} \sum_{i=1}^{k} \sum_{j-1}^{n_{i}}\left\{\log \left(\lambda_{i}\right)-\frac{\lambda_{i}\left(y_{i j}-\mu_{i}\right)^{2}}{\mu_{i}^{2} y_{i j}}\right\}
$$

Where $\theta=\left(\mu_{1}, \ldots . ., \mu_{k}, \lambda_{1}, \ldots ., \lambda_{k}\right)^{T}$. The overall maximum likelihood estimate is

$$
\hat{\theta}=\left(\bar{y}_{1}, \ldots \ldots, \bar{y}_{k}, v_{1}, \ldots ., v_{k}\right)^{T}
$$

Where

$$
\bar{y}_{i}=\frac{1}{n_{i}} \sum_{j=1}^{n i} y_{i j} \text { and } v_{i}=n_{i}\left[\sum_{j=1}^{n i}\left(\frac{1}{y_{i j}}-\frac{1}{\bar{y}_{1}}\right)\right]^{-1} \text {. }
$$

Furthermore, under the null hypothesis stated in (12) that the scale-like parameters are identical, the constrained maximum likelihood estimate is

$$
\tilde{\theta}=\left(\bar{y}_{1}, \ldots ., \bar{y}_{k}, v, \ldots, v\right)^{T}
$$

where

$$
v=\left[\sum_{i=1}^{k} n_{i}\right]\left[\sum_{i=1}^{k} \sum_{j=1}^{n_{i}}\left(\frac{1}{y_{i j}}-\frac{1}{\bar{y}_{i}}\right)\right]^{-1}
$$

Note that the model belongs to the exponential family model with canonical parameter $\varphi=\left(\varphi_{1}, \ldots . \varphi_{2 k}\right)^{T}$

where

$$
\varphi_{i}(\theta)=\left\{\begin{array}{l}
\lambda_{i} / 2 \mu_{i}^{2} i=1, \ldots ., k \\
\lambda_{i} / 2 i=1, \ldots ., k
\end{array}\right.
$$

And the corresponding sufficient statistic is $u=\left(u_{1}, \ldots . u_{2 k}\right)^{T}$ where

$$
u_{i}=\left\{\begin{array}{l}
n_{i} \bar{y}_{i} i=1, \ldots ., k \\
\sum_{i=1}^{n_{i}} 1 / y_{i j} i=1, \ldots ., k
\end{array}\right.
$$

The log-likelihood function expressed in terms of the canonical parameter and the sufficient statistic is

$$
l(\theta)=l(\varphi)=-\sum_{i=1}^{k}\left\{\varphi_{i} u_{i}=\varphi_{k+i} u_{k+1}-\frac{n_{i}}{2} \log \left(2 \varphi_{k+1}\right)-2 n_{i} \sqrt{\varphi_{k+1} \varphi_{i}}\right\} .
$$

By differentiating $l(\varphi)$ with respect $\varphi$, we have

$$
\begin{aligned}
& l_{\varphi i}(\varphi)=-u_{i}+n_{i} \sqrt{\varphi_{k+} / \varphi_{i}} \\
& l_{\varphi k+i}(\varphi)=-u_{k+i}+n_{i} \sqrt{\varphi_{i} / \varphi_{k+1}}+n_{i} / 2 \varphi_{k+1}
\end{aligned}
$$

for $i=1, \cdots, k$. Hence, the overall maximum likelihood estimate and the constrained maximum likelihood estimate of the canonical parameter are

$$
\begin{gathered}
\hat{\varphi}=\varphi(\hat{\theta})=\left(\frac{v_{1}}{2 \bar{y}_{1}^{2}}, \cdots, \frac{v_{k}}{2 \bar{y}_{k}^{2}}, \cdots, \frac{v_{k}}{2}\right)^{T} \\
\tilde{\varphi}=\left(\frac{v_{1}}{2 \bar{y}_{1}^{2}}, \cdots, \frac{v_{k}}{2 \bar{y}_{k}^{2}}, \frac{v}{2}, \cdots, \frac{v}{2}\right)^{T}
\end{gathered}
$$

Respectively. Moreover, let $\psi=\left(\psi_{1}, \ldots ., \psi_{k-1}\right)^{T} \quad$ where $\psi_{i}=\varphi_{k+1+i}-\varphi_{k+i}$. Then the hypothesis stated in (12) for testing homogeneity of the scale-like parameters can be expressed in terms of $\psi$ by

$$
H_{0}: \psi=0 \text { vs } H_{a}: \psi \neq 0 \text {. }
$$

Following the notation in Section 2, we have $\psi_{0}=0$, and $s_{\varphi}=-l_{\varphi}^{0}\left(\tilde{\varphi}^{0}\right)=\left[0, \cdots, 0, n_{1}\left(1 / v^{0}-1 / v_{1}^{0}\right), \cdots, n_{k}\left(1 / v^{0}-1 / v_{k}^{0}\right)\right]^{T}$.

Thus the log-likelihood function along the line $s(t)=t s^{0}+(1-t) s_{\varphi}$ is $l(\varphi)=\sum_{i=1}^{k}\left\{\varphi_{i} u_{i}+\varphi_{k+1}\left[u_{k+1}+n_{1}(1-t)\left(\frac{1}{v}-\frac{1}{v_{i}}\right)\right]-\frac{n_{i}}{2} \log \left(2 \varphi_{k+1}\right)-2 n_{i} \sqrt{\varphi_{k+i} \varphi_{i}}\right\}$,

Which depends on the value $t$ and is maximized at

$$
\begin{aligned}
& \hat{\varphi}_{i}=\left\{2 \bar{y}_{i}^{2}\left(\frac{1-t}{v}+\frac{t}{v_{i}}\right)\right\}^{-1} \\
& \hat{\varphi}_{k+i}=\left\{2\left(\frac{1-t}{v}+\frac{t}{v_{i}}\right)\right\}^{-1}
\end{aligned}
$$


For $i=1, \cdots, k$. It is easy to show that when $t=0$ and $t=1$, the maximize gives (16) and (15) respectively. Moreover, denote $t_{\max }=\frac{v_{(n)}}{v_{(n)}-v}$, where $v_{(n)}=\max \left(v_{1}, \cdots, v_{n}\right), \quad$ is the largest value of which the maximum likelihood estimator corresponding $t$ to $s(t)$ exists. thus, by substituting all the necessary terms into (9) and we have

$$
h(t ; \psi=0) \alpha \prod_{i=1}^{k}\left(\frac{1-t}{v}+\frac{t}{v_{i}}\right)^{\frac{n_{i}-3}{2}} .
$$

Finally, the directional p-value for testing homogeneity of the scale-like parameters can be computed from (11) with $\mathrm{h}(\mathrm{t})$ being defined in (18). Although there is no closed form for the integral, it requires only a one dimensional integral. Therefore, numerically, it can be easily obtained by some standard numerical integration routines such as those given in Maple, Matlab and R.

\section{Numerical examples}

In this section, we will first examine a real-life data example discussed in Kumagai \& Matsunaga [22], which is a time series data set of chlorobenzene exposure concentrations (15 min TWAs) for a chemical worker ( $\backslash$ Worker F" in the original paper) during a full workshift. The q-q plot in Takagi et al. [18] indicated that the q-q plot bin Takagi et al. [18] indicated that the $I G(\mu, \lambda)$ model is a reasonable model for analyzing this data set. Chang et al. [20] partitioned the data into the morning data and the afternoon data: (Table ) and assumed that the morning data and the afternoon data are samples from two independent $I G\left(\mu_{1}, \lambda_{1}\right)$ and $I G\left(\mu_{2}, \lambda_{2}\right)$ distributions. Table 1 records the p-values obtained for testing

Table 1: $p$-values for testing.

\begin{tabular}{|c|c|}
\hline Method & p-value \\
\hline Ir & 0.1045 \\
\hline LR & 0.1105 \\
\hline BN & 0.1133 \\
\hline
\end{tabular}

\begin{tabular}{|c|c|}
\hline Proposed & 0.1104 \\
\hline$H_{0}: \lambda_{1}=\lambda_{2}$ vs $H_{a}: \lambda_{1} \neq \lambda_{2}$
\end{tabular}

From the likelihood ratio method (lr) discussed in Chang et al. [20] , the Lugannani \& Rice method [5] (LR) and the BarndorffNielsen[6] modified signed likelihood ratio method (BN) discussed in Wong [21] and the directional method (Proposed) discussed in the paper. (Table 1)

$$
H_{0}: \lambda_{1}=\lambda_{2}
$$

The methods discussed in Wong [21] have theoretical accuracy of $O\left(n^{-3 / 2}\right)$. The proposed

Directional method give very similar result to that obtained by the Luggannani \& Rice method [5]. Result from likelihood ratio method, which has theoretical accuracy of $o\left({ }_{n}^{-1 / 2}\right)$, is not as close.

To compare the accuracy of the four methods, simulation studies were carried out. Following the set up in Chang et al. (2012), 5,000 samples are simulated for each settings of $\left(n_{1}, \cdots, n_{k}\right)$ and $\left(\mu_{1}, \cdots, \mu_{k} ; \lambda_{1}, \cdots, \lambda_{k}\right)$ with $\lambda_{1}=\cdots=\lambda_{k}=\lambda$. As in Chang et al. [19], $\mu_{1}, \cdots, \mu_{k}$ are all assumed to be 1 . For each for each simulated sample, we compute the p-values using lr, LR, BN and Proposed methods. The simulated Type I error probability is the proportion of $\mathrm{p}$-values less than the nominal significance level, which is chosen to be 0.05 and 0.1 respectively. The results are recorded in Table 2. It is clear that the lr method does not give satisfactory results unless $\left(n_{1}, \cdots, n_{k}\right)$ are large and the other three methods, regardless of the values of $, n_{1}, \cdots, n_{k}$ give very similar type I error probabilities and they are close to the nominal significance level. More simulations with different parameter settings have been performed with the same pattern of results. They are not reported here, but are available on request.

\begin{tabular}{|c|c|c|c|c|c|c|c|c|}
\hline & \multicolumn{4}{|c|}{$=0: 05$} & \multicolumn{4}{|c|}{$=0: 1$} \\
\hline & lr & LR & BN & Proposed & Ir & LR & BN & Proposed \\
\hline \multicolumn{9}{|c|}{$=(0: 3 ; 0: 3 ; 0: 3)$} \\
\hline $\mathrm{n}=(5 ; 5 ; 5)$ & 0.073 & 0.056 & 0.056 & 0.057 & 0.128 & 0.101 & 0.101 & 0.105 \\
\hline $\mathrm{n}=(5 ; 8 ; 10)$ & 0.061 & 0.051 & 0.051 & 0.048 & 0.113 & 0.096 & 0.095 & 0.098 \\
\hline $\mathrm{n}=(10 ; 10 ; 10)$ & 0.055 & 0.048 & 0.048 & 0.049 & 0.118 & 0.105 & 0.102 & 0.106 \\
\hline $\mathrm{n}=(10 ; 15 ; 20)$ & 0.064 & 0.056 & 0.056 & 0.056 & 0.111 & 0.103 & 0.103 & 0.102 \\
\hline $\mathrm{n}=(30 ; 30 ; 30)$ & 0.05 & 0.046 & 0.046 & 0.048 & 0.102 & 0.099 & 0.099 & 0.1 \\
\hline \multicolumn{9}{|c|}{$=(0: 5 ; 0: 5 ; 0: 5 ; 0: 5 ; 0: 5)$} \\
\hline $\mathrm{n}=(5 ; 5 ; 5 ; 5 ; 5)$ & 0.074 & 0.055 & 0.055 & 0.052 & 0.135 & 0.104 & 0.104 & 0.103 \\
\hline $\mathrm{n}=(7 ; 8 ; 9 ; 10 ; 12)$ & 0.061 & 0.05 & 0.05 & 0.051 & 0.114 & 0.1 & 0.1 & 0.101 \\
\hline $\mathrm{n}=(10 ; 10 ; 10 ; 10 ; 10)$ & 0.064 & 0.053 & 0.053 & 0.054 & 0.115 & 0.101 & 0.101 & 0.1 \\
\hline $\mathrm{n}=(12 ; 15 ; 16 ; 18 ; 20)$ & 0.054 & 0.048 & 0.048 & 0.05 & 0.109 & 0.1 & 0.1 & 0.102 \\
\hline $\mathrm{n}=(20 ; 20 ; 30 ; 30 ; 30)$ & 0.049 & 0.047 & 0.046 & 0.047 & 0.102 & 0.096 & 0.095 & 0.096 \\
\hline
\end{tabular}

Table 2 : Simulated type I error (based on 5,000 simulations). 


\section{Biostatistics and Biometrics Open Access Journal}

\begin{tabular}{|c|c|c|c|c|c|c|c|c|}
\hline \multicolumn{7}{|c|}{$=(1 ; 1 ; 1 ; 1 ; 1 ; 1 ; 1 ; 1)$} \\
\hline $\mathrm{n}=(5 ; 5 ; 5 ; 5 ; 5 ; 5 ; 5 ; 5)$ & 0.082 & 0.055 & 0.054 & 0.055 & 0.143 & 0.109 & 0.109 & 0.108 \\
\hline $\mathrm{n}=(7 ; 8 ; 10 ; 12 ; 12 ; 14 ; 14 ; 15)$ & 0.072 & 0.059 & 0.059 & 0.06 & 0.128 & 0.114 & 0.114 & 0.115 \\
\hline $\mathrm{n}=(10 ; 10 ; 10 ; 10 ; 10 ; 10 ; 10 ; 10)$ & 0.061 & 0.05 & 0.05 & 0.051 & 0.119 & 0.101 & 0.101 & 0.103 \\
\hline $\mathrm{n}=(10 ; 12 ; 15 ; 15 ; 18 ; 18 ; 20 ; 20)$ & 0.06 & 0.053 & 0.053 & 0.051 & 0.116 & 0.104 & 0.103 & 0.106 \\
\hline $\mathrm{n}=(20 ; 20 ; 30 ; 30 ; 30 ; 30 ; 40 ; 40)$ & 0.061 & 0.057 & 0.057 & 0.056 & 0.113 & 0.105 & 0.105 & 0.106 \\
\hline \multicolumn{7}{|c|}{$=(1: 2 ; 1: 2 ; 1: 2 ; 1: 2 ; 1: 2 ; 1: 2 ; 1: 2 ; 1: 2 ; 1: 2 ; 1: 2)$} & & 0.105 \\
\hline $\mathrm{n}=(5 ; 5 ; 5 ; 5 ; 5 ; 5 ; 5 ; 5 ; 5 ; 5)$ & 0.079 & 0.052 & 0.052 & 0.056 & 0.146 & 0.102 & 0.102 & 0.105 \\
\hline $\mathrm{n}=(7 ; 8 ; 8 ; 10 ; 10 ; 12 ; 12 ; 12 ; 15 ;$ & 0.061 & 0.047 & 0.047 & 0.053 & 0.119 & 0.103 & 0.103 & 0.105 \\
\hline $\mathrm{n}=(10 ; 10 ; 10 ; 10 ; 10 ; 10 ; 10 ; 10 ;$ \\
$10 ; 10)$
\end{tabular}

\section{Discussion}

A directional test for a vector parameter of interest from a natural exponential family model is derived based on the likelihood-based higher order asymptotic methods. The p-value for the test required only a one-dimensional integration. The proposed method is then applied to obtain p-value for testing homogeneity of inverse Gaussian scale-like parameters. Numerical results indicated that the proposed methods are as accurate as the Lugannani and Rice method and the BarndorNielsen method, which are third-order asymptotic methods.

\section{References}

1. Lehmann EL, Romano JP (2005) Testing Statistical Hypotheses. Springer New York, USA.

2. DiCiccio TJ, Field CA, Fraser DAS (1990) Approximations of marginal tail probabilities and inference for scalar parameters. Biometrika 77: 77-95.

3. Reid N (1996) Likelihood and higher-order approximations to tail areas: a review and annotated bibliography. Canadian Journal of Statistics 24(2): 141-166

4. Daniels HE (1954) Saddlepoint approximation in statistics. Ann Math Stat 25: 631-650.

5. Lugannani, R. and Rice, S., 1980. Saddlepoint approximation for the distribution of the sum of independent random variables. Adv in Appl Probab 12: 475-490.

6. Barndorff -Nielsen, OE, 1986. Inference on full or partial parameters based on the stan-dardized signed log likelihood ratio. Biometrika 73: 307-322.

7. Barndorff-Nielsen, OE (1991) Modified signed log likelihood ratio. Biometrika 78: 557-561.

8. Brazzale AR, Davison AC, Reid N(2007) Applied Asymptotics. Cambridge: Cam-bridge University Press, USA.
9. Skovgaard IM (2001) Likelihood asymptotics. Scandinavian Journal of Statistics 28: 3-32.

10. Fraser DAS, Massam H (1985) Conical tests: observed level of significance and confidence regions. Statistics Hefte 26(1): 1-17.

11. Davison AC (1988) Approximate conditional inference in generalized linear models. Journal of the Royal Statistical Society B 50: 445-461.

12. Davison AC, Fraser DAS, Reid N, Sartori N (2014) Accurate directional inference for vector parameters in linear exponential families. Journal of the American Statistical Association 109: 302-314

13. Fraser DAS, Reid N, Wong A (1991) Exponential linear models: a two pass procedure for saddle point approximation. Journal of the Royal Statistical Society B 53: 483-492.

14. Skovgaard IM (1987) Saddlepoint expansions for conditional distributions. Journal of Applied Probability, 875-887.

15. Doksum KA, Hoyland A (1992) Models for variable-stress accelerated life testing experiments based on Wiener processes and the inverse Gaussian. Technometrics 34: 74-82.

16. Seshadri V (1993) The Inverse Gaussian Distribution: A Case Study in Exponential Families. Claredon Press, Oxford.

17. Seshadri V (1999) The Inverse Gaussian Distribution: Statistical Theory and Applications. Springer, New York, USA.

18. Takagi K, Kumagai S, Matsunaga I, Kusaka Y (1997) Application of inverse Gaussian distribution to occupational exposure data. Annals of Occupational Hygiene 41: 505-514.

19. Chhikara, R.S. and Folks, J.L., 1989. The Inverse Gaussian Distribution Marcel Dekker, New York.

20. Chang M, You X, Wen M (2012) Testing the homogeneity of inverse Gaussian scale-like parameters. Stat. Probab Lett 82: 1755-1760.

21. Wong A (2016) Testing homogeneity of inverse Gaussian scale-like parameters: a saddlepoint approach. Statistical Papers 57(2): 319-327.

22. Kumagai S, Matsunaga I (1995) Changes in the distribution of shortterm exposure concentration with different averaging times. Am Ind Hyg Assoc J 56(1): 24-31. 
This work is licensed under Creative Commons Attribution 4.0 Licens

DOI: 10.19080/BBOAJ.2017.03.555608
Your next submission with Juniper Publishers will reach you the below assets

- Quality Editorial service

- Swift Peer Review

- Reprints availability

- E-prints Service

- Manuscript Podcast for convenient understanding

- Global attainment for your research

- Manuscript accessibility in different formats

( Pdf, E-pub, Full Text, Audio)

- Unceasing customer service

Track the below URL for one-step submission https://juniperpublishers.com/online-submission.php 